\title{
Uniqueness and Existence of the Integrated Density of States for Schrödinger Operators with Magnetic Field and Electric Potential with Singular Negative Part
}

By

Viorel IfTimiE*

\begin{abstract}
We prove the coincidence of the two definitions of the integrated density of states (IDS) for Schrödinger operators with strongly singular magnetic fields and scalar potentials: the first one using the counting function of eigenvalues of the induced operator on a bounded open set with Dirichlet boundary conditions, the second one using the spectral projections of the whole space operator. Thus we generalize a result of [5], where the scalar potential was non-negative. Moreover, we prove the existence of IDS for the case of periodical magnetic field and scalar potential.
\end{abstract}

\section{$\S 1$. Introduction}

One considers the vector potential $a=\left(a_{1}, \ldots, a_{n}\right): \mathbb{R}^{n} \rightarrow \mathbb{R}^{n}, n \geq 2$ (which is identified to the differential form $\left.\sum_{1 \leq j \leq n} a_{j} \mathrm{~d} x_{j}\right)$ and the scalar potential $V: \mathbb{R}^{n} \rightarrow \mathbb{R}$ satisfying the following hypotheses:

i) $a_{j} \in L_{\text {loc }}^{2}\left(\mathbb{R}^{n}\right), 1 \leq j \leq n$;

ii) $V \in L_{\text {loc }}^{1}\left(\mathbb{R}^{n}\right)$ and $V_{-}:=\max (0,-V)$ belongs to the Kato class $K_{n}$, that is, one has:

Communicated by T. Kawai. Received March 19, 2003.

2000 Mathematics Subject Classification(s): 35P05, 35J10, 47F05.

*University of Bucharest, Faculty of Mathematics, 14 Academiei Street, Bucharest, 70109, Romania.

(C) 2005 Research Institute for Mathematical Sciences, Kyoto University. All rights reserved. 


$$
\lim _{\varepsilon \searrow 0}\left[\sup _{x \in \mathbb{R}^{n}} \int_{|x-y|<\varepsilon} E(x-y) V_{-}(y) \mathrm{d} y\right]=0,
$$

where $E$ is the usual elementary solution of the Laplace operator $\Delta$.

We define the sesqui-linear form $h=h(a, V)$ on $L^{2}\left(\mathbb{R}^{n}\right)$ with domain

$$
D(h)=\left\{u \in L^{2}\left(\mathbb{R}^{n}\right) ;(\nabla-\mathrm{i} a) u \in\left(L^{2}\left(\mathbb{R}^{n}\right)\right)^{n},|V|^{1 / 2} u \in L^{2}\left(\mathbb{R}^{n}\right)\right\},
$$

by

$$
h(u, v)=\int_{\mathbb{R}^{n}}(\nabla-\mathrm{i} a) u \cdot \overline{(\nabla-\mathrm{i} a) v} \mathrm{~d} x+\int_{\mathbb{R}^{n}} V u \bar{v} \mathrm{~d} x,
$$

where $\nabla$ stands for the distributional gradient and $\mathrm{i}=\sqrt{-1}$.

It is well-known (see [14]) that $h$ is bounded from below and closed, the space $\mathcal{C}_{0}^{\infty}\left(\mathbb{R}^{n}\right)$ being a core of $h$. Let $H=H(a, V)$ be the associated self-adjoint bounded from below operator on $L^{2}\left(\mathbb{R}^{n}\right)$, with domain

$$
D(H)=\left\{u \in D(h) ;-(\nabla-\mathrm{i} a)^{2} u+V u \in L^{2}\left(\mathbb{R}^{n}\right)\right\},
$$

given by

$$
H u=-(\nabla-\mathrm{i} a)^{2} u+V u .
$$

We shall also need a self-adjoint realization of the differential operator $-(\nabla-$ i $a)^{2}+V$ on a open subset $\Omega$ of $\mathbb{R}^{n}$, corresponding to the Dirichlet boundary conditions. One identifies $L^{2}(\Omega)$ to the closed subspace of $L^{2}\left(\mathbb{R}^{n}\right)$ with elements which are zero on $\mathbb{R}^{n} \backslash \Omega$. Let $P_{\Omega}$ be the projection of $L^{2}\left(\mathbb{R}^{n}\right)$ onto $L^{2}(\Omega)$ (the multiplication operator by the characteristic function of $\Omega$ ). If $H_{\alpha}:=$ $H+\alpha\left(1-P_{\Omega}\right), \alpha \geq 0$, one obtains an unique operator $H_{\Omega}$, pseudo-selfadjoint on $L^{2}\left(\mathbb{R}^{n}\right)$, such that $\lim _{\alpha \rightarrow \infty} H_{\alpha}=H_{\Omega}$ in the strong resolvent sense (see Th. 4.1 in [10]). Moreover, the operator $H_{\Omega}$ can be considered as a self-adjoint operator on $L^{2}(\Omega)$ associated with the sesqui-linear form $h_{\Omega}$ defined by:

$$
h_{\Omega}(u, v):=h(u, v), \quad D\left(h_{\Omega}\right):=\{u \in D(h) ; \operatorname{supp} u \subset \bar{\Omega}\},
$$

identified to a form on $L^{2}(\Omega)$.

Remark 1.1. Usually, one works with another Dirichlet realization on $\Omega$ (see [5], for instance). More exactly, one considers the operator $\tilde{H}_{\Omega}$, self-adjoint on $L^{2}(\Omega)$, associated with the sesqui-linear form $\tilde{h}_{\Omega}$, which is the closure on $L^{2}(\Omega)$ of the form $h_{\Omega}^{\circ}$ with domain $\mathcal{C}_{0}^{\infty}(\Omega)$, defined by

$$
h_{\Omega}^{\circ}(u, v)=\int_{\Omega}(\nabla-\mathrm{i} a) u \cdot \overline{(\nabla-\mathrm{i} a) v} \mathrm{~d} x+\int_{\Omega} V u \bar{v} \mathrm{~d} x .
$$


We shall see in $\S 3$ that $H_{\Omega}=\tilde{H}_{\Omega}$ if $\Omega$ is a "Lipschitz domain" (or, following Stein [15], a domain with "minimally smooth boundary").

In order to state the main result, we shall need a family $\mathcal{F}$ of bounded open subsets of $\mathbb{R}^{n}$, satisfying the conditions:

iii) For every $m \in \mathbb{N}^{*}$, there exists $\Omega \in \mathcal{F}$ such that the ball $B(0 ; m)$, with centre at the origin and of radius $m$, is contained in $\Omega$.

iv) For every $\varepsilon>0$, there exists $m_{0} \in \mathbb{N}^{*}$ such that if $\Omega \in \mathcal{F}$ and $B\left(0 ; m_{0}\right) \subset \Omega$, one has

$$
|\{x \in \Omega ; \operatorname{dist}(x, \partial \Omega)<1\}|<\varepsilon|\Omega| .
$$

Definition 1.2. Let $\mu, \mu_{\Omega}(\Omega \in \mathcal{F})$ be Borel measures on $\mathbb{R}$. We say that

$$
\lim _{\Omega \rightarrow \mathbb{R}^{n}, \Omega \in \mathcal{F}} \mu_{\Omega}=\mu,
$$

if for every $f \in \mathcal{C}_{0}(\mathbb{R})$ (the space of compactly supported continuous functions on $\mathbb{R})$ and for every $\varepsilon>0$, there exists $m_{0} \in \mathbb{N}^{*}$ such that if $B\left(0 ; m_{0}\right) \subset \Omega$, than one has

$$
\left|\int_{\mathbb{R}} f \mathrm{~d} \mu_{\Omega}-\int_{\mathbb{R}} f \mathrm{~d} \mu\right|<\varepsilon .
$$

We shall see that for every $f \in \mathcal{C}_{0}(\mathbb{R})$ and for every $\Omega$ bounded open subset of $\mathbb{R}^{n}$, the operators $f\left(H_{\Omega}\right)$ and $P_{\Omega} f(H) P_{\Omega}$ belong to $\mathcal{I}_{1}$ (the space of trace class operators). Then, using the Riesz-Markov Theorem, there exist Borel measures $\mu_{\Omega}^{D}$ and $\mu_{\Omega}$, such that

$$
|\Omega|^{-1} \operatorname{Tr} f\left(H_{\Omega}\right)=\int_{\mathbb{R}} f \mathrm{~d} \mu_{\Omega}^{D}, \quad|\Omega|^{-1} \operatorname{Tr}\left(P_{\Omega} f(H) P_{\Omega}\right)=\int_{\mathbb{R}} f \mathrm{~d} \mu_{\Omega} .
$$

One sees that the distribution functions of those two measures satisfy the relations

$$
\mu_{\Omega}^{D}((-\infty, \lambda])=|\Omega|^{-1} N_{\Omega}(\lambda), \quad \mu_{\Omega}((-\infty, \lambda])=|\Omega|^{-1} \operatorname{Tr}\left(P_{\Omega} E_{\lambda}(H) P_{\Omega}\right),
$$

almost everywhere on $\mathbb{R}$, where $N_{\Omega}(\lambda)$ is the number of the eigenvalues of $H_{\Omega}$ which are less than $\lambda$, and $E_{\lambda}(H)$ is the spectral projection of $H$ for the interval $(-\infty, \lambda], \lambda \in \mathbb{R}$.

We can define the integrated density of states in two different ways. 
Definition 1.3. We call density of states of $H$ a Borel measure $\mu^{D}$ (respectively $\mu$ ) on $\mathbb{R}$ such that

$$
\lim _{\Omega \rightarrow \mathbb{R}^{n}, \Omega \in \mathcal{F}} \mu_{\Omega}^{D}=\mu^{D} \quad\left(\text { respectively } \lim _{\Omega \rightarrow \mathbb{R}^{n}, \Omega \in \mathcal{F}} \mu_{\Omega}=\mu\right) .
$$

The distribution function $\rho^{D}$ of $\mu^{D}$ (respectively $\rho$ of $\mu$ ) will be the integrated density of states of $H$.

This definition rises two problems:

a) Prove the equivalence of the two definitions of IDS.

b) Prove the existence of IDS.

The solution of problem a) is the main result of this paper:

Theorem 1.4. Under hypotheses i)-iv), the density of states $\mu^{D}$ exists if and only if the density of states $\mu$ exists. Moreover, if one of them exists, then $\mu^{D}=\mu$.

This theorem was proved in [5] in the case where $V \geq 0$. The proof in $\S 4$ uses some ideas of [5], along with a property of comparison of resolvents, essentially proved in [4], and which requires the hypothesis $V_{-} \in K_{n}$.

Remark 1.5. An analysis of the proof of Theorem 1.2 in [5] shows that if $\mathcal{F} \subset \operatorname{LM}(r, A, B)$ (see the notation in [5]), Theorem 1.4 remains true if the Dirichlet boundary conditions are replaced by Neumann boundary conditions.

The problem b) will be solved only in a special case. Let

$$
B=\mathrm{d} a=\frac{1}{2} \sum_{1 \leq j, k \leq n} B_{j k} \mathrm{~d} x_{j} \wedge \mathrm{d} x_{k}, \quad B_{j k}=\partial_{j} a_{k}-\partial_{k} a_{j}
$$

be the magnetic field defined by the vector potential $a$. ( $B_{j k}$ will be distributions on $\mathbb{R}^{n}$.) One considers a lattice $\Gamma$ in $\mathbb{R}^{n}$, generated by a basis $e_{1}, \ldots, e_{n}$, that is,

$$
\Gamma=\left\{\sum_{j=1}^{n} \alpha_{j} e_{j} ; \alpha_{j} \in \mathbb{Z}, 1 \leq j \leq n\right\} .
$$

One denotes by $F$ a fundamental domain of $\mathbb{R}^{n}$ with respect to $\Gamma$; for instance,

$$
F=\left\{\sum_{j=1}^{n} t_{j} e_{j} ; 0 \leq t_{j}<1,1 \leq j \leq n\right\} .
$$


We also remark that for every $f \in \mathcal{C}_{0}(\mathbb{R})$ and every $\Omega$ bounded open subset of $\mathbb{R}^{n}, P_{\Omega} f(h) P_{\Omega}$ is the product of two operators from $\mathcal{I}_{2}$ (the space of HilbertSchmidt operators). By the Fubini Theorem, the restriction of the integral kernel $K_{f(H)}$ of $f(H)$ to the diagonal set of $\mathbb{R}^{n} \times \mathbb{R}^{n}$ is well-defined and is a locally integrable function.

We suppose that the following two hypotheses hold:

iv') For every $\varepsilon>0$ there exists $m_{0} \in \mathbb{N}^{*}$ such that, if $\Omega \in \mathcal{F}$ and $B\left(0 ; m_{0}\right) \subset \Omega$, then one has

$$
\left|\left\{x \in \mathbb{R}^{n} ; \operatorname{dist}(x, \partial \Omega)<1\right\}<\varepsilon\right| \Omega \mid .
$$

v) $V$ and $B_{j k}, 1 \leq j, k \leq n$ are $\Gamma$-periodic functions.

Theorem 1.6. Under hypotheses i)-iii), iv') and v), the IDS of $H$ exists and, for every $f \in \mathcal{C}_{0}(\mathbb{R})$, one has

$$
\lim _{\Omega \rightarrow \mathbb{R}^{n}, \Omega \in \mathcal{F}} \frac{\operatorname{Tr}\left(P_{\Omega} f(H) P_{\Omega}\right)}{|\Omega|}=\frac{1}{|F|} \int_{F} K_{f(H)}(x, x) \mathrm{d} x .
$$

We shall see that the integral above represents a $\Gamma$-trace of the operator $f(H)$, in the sense of Atiyah [1].

The theorem above is known in the case where $B$ is a constant magnetic field and $V \in \mathcal{C}^{\infty}\left(\mathbb{R}^{n}\right)$ (see [6]). The case of constant magnetic fields and random electric potentials, possibly unbounded from below, was also studied (see [7]).

The plan of the paper is the following: In the second section we prove some properties of the operator $H$. Particularly, for the reader convenience, we give the proof of the property of comparison of resolvents. The third section is devoted to the study of the operator $H_{\Omega}$ : we prove the identity $H_{\Omega}=\tilde{H}_{\Omega}$ for domains with minimally smooth boundary and we generalize the aforementioned property of comparison to this case. In the last two sections we prove Theorems 1.4 and 1.6, respectively.

\section{$\S 2$. The Operator $H=H(a, V)$}

Proposition 2.1. $\quad$ Under hypotheses i) and ii), for every $\rho>1$, there exist $M, \delta>0$ such that if $\lambda>\max \{\delta,-\inf \sigma(H)\}($ where $\sigma(H)$ is the spectrum of $H)$, then one has

$$
\left|(H+\lambda)^{-r} f\right| \leq M\left(\rho H_{0}+\lambda-\delta\right)^{-r}|f| \quad \text { a.e. on } \mathbb{R}^{n}
$$

for every $r>0$ and $f \in L^{2}\left(\mathbb{R}^{n}\right)$, where $H_{0}:=H(0,0)$. 
Proof. Firstly, one remarks that, following [2], for every $f \in L^{2}\left(\mathbb{R}^{n}\right)$ and $t>0$, one has the inequality

$$
\left|\mathrm{e}^{-t H(a, V)} f\right| \leq \mathrm{e}^{-t H(0, V)}|f|, \quad \text { a.e on } \mathbb{R}^{n} .
$$

Using the Feymann-Kaç formula (see, for instance, [13]), we infer that

$$
\mathrm{e}^{-t H(0, V)}|f| \leq \mathrm{e}^{-t H\left(0,-V_{-}\right)}|f| \text { a.e on } \mathbb{R}^{n} .
$$

It is known (see Proposition B.6.7 in [14]) that $\mathrm{e}^{-t H\left(0,-V_{-}\right)}$is an integral operator whose integral kernel $k: \mathbb{R}_{+}^{*} \times \mathbb{R}^{n} \times \mathbb{R}^{n} \rightarrow \mathbb{R}_{+}^{*}$ is a continuous function which verifies the following estimate: for every $\rho>1$, there exist the positive constants $M$ and $\delta$ such that, for every $t>0$ and $x, y \in \mathbb{R}^{n}$, one has

$$
|k(t, x, y)| \leq M \mathrm{e}^{\delta t} k_{0}(\rho t, x, y),
$$

where $k_{0}$ is the integral kernel of $\mathrm{e}^{-t H_{0}}$.

We also have

$$
(H+\lambda)^{-r} f=\frac{1}{\Gamma(r)} \int_{0}^{\infty} t^{r-1} \mathrm{e}^{-\lambda t} \mathrm{e}^{-t H} f \mathrm{~d} t,
$$

for every $r>0, \lambda>-\inf \sigma(H)$ and $f \in L^{2}\left(\mathbb{R}^{n}\right)$, where $\Gamma(\cdot)$ is the Euler gamma function.

If $\lambda>\max \{\delta,-\inf \sigma(H)\}$, the relations (2.2)-(2.5) imply the inequalities

$$
\begin{aligned}
\left|(H+\lambda)^{-r} f\right| & \leq \frac{M}{\Gamma(r)} \int_{0}^{\infty} t^{r-1} \mathrm{e}^{-(\lambda-\delta) t} \mathrm{e}^{-\rho t H_{0}}|f| \mathrm{d} t \\
& =M\left(\rho H_{0}+\lambda-\delta\right)^{-r}|f| \text { a.e. on } \mathbb{R}^{n},
\end{aligned}
$$

which finish the proof.

Remark 2.2. For the case $V \geq 0$, one proves in [9] that for $\lambda>0$ and $f \in L^{2}\left(\mathbb{R}^{n}\right)$ one has

$$
\left|(H+\lambda)^{-1} f\right| \leq\left(H_{0}+\lambda\right)^{-1}|f|, \quad \text { a.e. on } \mathbb{R}^{n} .
$$

It is this equality (or rather an extension of it at $H_{\Omega}$ ) which is used in [5].

Remark 2.3. In [4], one gives an example which shows that the hypothesis $V_{-} \in K_{n}$ is necessary for the validity of inequality (2.1) 
Proposition 2.4. Under the hypotheses of Proposition 2.1, for every $r>n / 4$ and $\lambda>\max \{\delta,-\inf \sigma(H)\}$, there exists a positive constant $C$ such that, for every open bounded subset $\Omega$ of $\mathbb{R}^{n}$, we have that $P_{\Omega}(H+\lambda)^{-r} \in \mathcal{I}_{2}$ and the inequality

$$
\left\|P_{\Omega}(H+\lambda)^{-r}\right\|_{\mathcal{I}_{2}} \leq C|\Omega|^{1 / 2}
$$

holds.

Proof. Using (2.1) we infer that for every $f \in L^{2}\left(\mathbb{R}^{n}\right)$,

$$
\left|P_{\Omega}(H+\lambda)^{-r} f\right| \leq M \rho^{-r} P_{\Omega}\left(H_{0}+\frac{\lambda-\delta}{\rho}\right)^{-r}|f| \text { a.e. on } \mathbb{R}^{n} .
$$

On the other hand, $\left(H_{0}+\frac{\lambda-\delta}{\rho}\right)^{-r}$ is a convolution operator by a function $g \in L^{2}\left(\mathbb{R}^{n}\right)$; therefore the operator in the right hand side of (2.7) belongs to $\mathcal{I}_{2}$, since its integral kernel is $M \rho^{-r} \chi_{\Omega}(x) g(x-y)$, where $\chi_{\Omega}$ is the characteristic function of $\Omega$. Furthermore,

$$
\left\|P_{\Omega}\left(H_{0}+\frac{\lambda-\delta}{\rho}\right)^{-r}\right\|_{\mathcal{I}_{2}}=\int_{\Omega} \int_{\mathbb{R}^{n}}|g(x-y)|^{2} \mathrm{~d} x \mathrm{~d} y=\|g\|_{L^{2}\left(\mathbb{R}^{n}\right)}^{2}|\Omega| .
$$

To obtain the stated properties, it suffices to use (2.7), (2.8) and Theorem 2.13 in $[12]$.

Corollary 2.5. Under the hypotheses of Proposition 2.1, for every $m>$ $n / 2$ and $\lambda>\max \{\delta,-\inf \sigma(H)\}$, there exists a positive constant $C$ such that, for every open bounded subset $\Omega$ of $\mathbb{R}^{n}$, we have that $P_{\Omega}(H+\lambda)^{-m} P_{\Omega} \in \mathcal{I}_{1}$ and the inequality

$$
\left\|P_{\Omega}(H+\lambda)^{-m} P_{\Omega}\right\|_{\mathcal{I}_{1}} \leq C|\Omega|
$$

holds.

Proof. It suffices to use Proposition 2.4 and the inequality

$$
\left\|P_{\Omega}(H+\lambda)^{-m} P_{\Omega}\right\|_{\mathcal{I}_{1}} \leq\left\|P_{\Omega}(H+\lambda)^{-r}\right\|_{\mathcal{I}_{2}} \cdot\left\|(H+\lambda)^{-s} P_{\Omega}\right\|_{\mathcal{I}_{2}},
$$

where $r, s>n / 4$ and $r+s=m$.

Corollary 2.6. $\quad$ For every $f \in L_{\text {comp }}^{\infty}(\mathbb{R})$, there exists a constant $C>0$, such that for every open bounded subset $\Omega$ of $\mathbb{R}^{n}$, we have that $P_{\Omega} f(H) P_{\Omega} \in \mathcal{I}_{1}$ and the inequality

$$
\left\|P_{\Omega} f(H) P_{\Omega}\right\|_{\mathcal{I}_{1}} \leq C|\Omega|
$$

holds. 
Proof. It suffices to write the identity

$$
P_{\Omega} f(H) P_{\Omega}=P_{\Omega}(H+\lambda)^{-r}(H+\lambda)^{2 r} f(H)(H+\lambda)^{-r} P_{\Omega},
$$

where $r>n / 4$ and $\lambda>\max \{\delta,-\inf \sigma(H)\}$ and to apply Proposition 2.4.

In order to state the last result of this section, we denote by $\mathcal{B}(E, F)$ the space of all bounded linear operators from $E$ to $F$ ( $E$ and $F$ being normed linear spaces). In particular, $\mathcal{B}(E):=\mathcal{B}(E, E)$.

Lemma 2.7. Let $\varphi \in \mathcal{C}^{\infty}\left(\mathbb{R}^{n}\right)$ be such that $\partial^{\alpha} \varphi \in L^{\infty}\left(\mathbb{R}^{n}\right)$ if $|\alpha| \leq 2$. Then $\varphi$ belongs to $D(H)$ for every $u \in D(H)$. Moreover,

$$
[H, \varphi]=-2(\nabla \varphi) \cdot(\nabla-\mathrm{i} a)-\Delta \varphi \quad \text { on } D(H)
$$

and

$$
(H+\lambda)^{-1}[H, \varphi] \in \mathcal{B}\left(L^{2}\left(\mathbb{R}^{n}\right)\right) \quad \text { if } \quad \lambda>-\inf \sigma(H) .
$$

Proof. If $u \in D(H)$, then $\varphi u \in D(h)$ and for every $v \in \mathcal{C}_{0}^{\infty}\left(\mathbb{R}^{n}\right)$,

$$
\begin{aligned}
h(\varphi u, v) & =h(u, \bar{\varphi} v)-2((\nabla \varphi) \cdot(\nabla-\mathrm{i} a) u, v)-((\Delta \varphi) u, v) \\
& =(\varphi H u-2(\nabla \varphi) \cdot(\nabla-\mathrm{i} a) u-(\Delta \varphi) u, v),
\end{aligned}
$$

where $(\cdot, \cdot)$ denotes the scalar product of $L^{2}\left(\mathbb{R}^{n}\right)$. We deduce that $\varphi u \in D(H)$ and

$$
H(\varphi u)=\varphi H u-2(\nabla \varphi) \cdot(\nabla-\mathrm{i} a) u-(\Delta \varphi) u .
$$

This yields (2.11). To get (2.12), we endow $D(H)$ with the graph topology. Then $[H, \varphi] \in \mathcal{B}\left(D(H), L^{2}\left(\mathbb{R}^{n}\right)\right)$ and, by duality, $[H, \varphi] \in \mathcal{B}\left(L^{2}\left(\mathbb{R}^{n}\right),[D(H)]^{*}\right)$, while $(H+\lambda)^{-1} \in \mathcal{B}\left(L^{2}\left(\mathbb{R}^{n}\right), D(H)\right)$ and $(H+\lambda)^{-1} \in \mathcal{B}\left([D(H)]^{*}, L^{2}\left(\mathbb{R}^{n}\right)\right)$ by duality.

\section{§3. The Operator $H_{\Omega}=H_{\Omega}(a, V)$.}

We fix $\gamma \in \mathbb{R}$ such that $h \geq \gamma$. Then $D(h)$ is a Hilbert space for the norm

$$
\|u\|_{h}=\left[h(u, u)+(\gamma+1)\|u\|^{2}\right]^{1 / 2}, \quad u \in D(h),
$$

where $\|\cdot\|$ denotes the norm of $L^{2}\left(\mathbb{R}^{n}\right)$.

Let $\mathcal{C}_{+\infty}(\mathbb{R}):=\left\{f \in \mathcal{C}(\mathbb{R}) ; \lim _{t \rightarrow+\infty} f(t)=0\right\}$. Then for $f \in \mathcal{C}_{+\infty}(\mathbb{R})$ and $\Omega$ open subset of $\mathbb{R}^{n}$, we can define $f\left(H_{\Omega}\right) \in \mathcal{B}\left(L^{2}\left(\mathbb{R}^{n}\right)\right)$ in the following way: $\left.f\left(H_{\Omega}\right)\right|_{L^{2}(\Omega)}$ is the operator from $\mathcal{B}\left(L^{2}(\Omega)\right)$ associated with $H_{\Omega}$, as selfadjoint operator on $L^{2}(\Omega)$, by the usual functional calculus, while $f\left(H_{\Omega}\right)=0$ on $L^{2}(\Omega)^{\perp}$. 
Proposition 3.1. Let $f \in \mathcal{C}_{+\infty}(\mathbb{R}), \lambda>-\inf \sigma(H)$ and $\varphi$ as in Lemma 2.7. Then, for every open set $\Omega \subset \mathbb{R}^{n}$, the operator $H_{\Omega}$ has the following properties:

a) $s-\lim _{\alpha \rightarrow \infty} f\left(H_{\alpha}\right)=f\left(H_{\Omega}\right)$ in $\mathcal{B}\left(L^{2}\left(\mathbb{R}^{n}\right)\right)$.

b) $s-\lim _{\alpha \rightarrow \infty}\left(H_{\alpha}+\lambda\right)^{-1}=\left(H_{\Omega}+\lambda\right)^{-1}$ in $\mathcal{B}\left([D(h)]^{*}, D(h)\right)$.

c) $\left(H_{\Omega}+\lambda\right)^{-1}=P_{\Omega}\left(H_{\Omega}+\lambda\right)^{-1}=\left(H_{\Omega}+\lambda\right)^{-1} P_{\Omega}$.

d) $\left(H_{\Omega}+\lambda\right)^{-1}[H, \varphi],[H, \varphi]\left(H_{\Omega}+\lambda\right)^{-1} \in \mathcal{B}\left(L^{2}\left(\mathbb{R}^{n}\right)\right)$.

e) $s-\lim _{\alpha \rightarrow \infty}[H, \varphi]\left(H_{\alpha}+\lambda\right)^{-1}=[H, \varphi]\left(H_{\Omega}+\lambda\right)^{-1}$ in $\mathcal{B}\left(L^{2}\left(\mathbb{R}^{n}\right)\right)$.

f) $\left(\partial_{k}-\mathrm{i} a_{k}\right)\left(H_{\Omega}+\lambda\right)^{-1} \in \mathcal{B}\left(L^{2}(\Omega)\right), 1 \leq k \leq n$.

Proof. a) The property follows from [3], §3.

b) The property is a consequence of Lemma 3.7 in [3].

c) By the inequality (3.7) in [3], there exists a constant $C>0$ such that, for every $\alpha>0$, we have

$$
\left\|\left(1-P_{\Omega}\right)\left(H_{\alpha}+\lambda\right)^{-1}\right\|_{\mathcal{B}\left(L^{2}\left(\mathbb{R}^{n}\right)\right)} \leq C \alpha^{-1 / 2},
$$

whence we get the needed inequalities.

d) The property follows from the fact that

$$
\left(H_{\Omega}+\lambda\right)^{-1} \in \mathcal{B}\left(L^{2}\left(\mathbb{R}^{n}\right), D(h)\right) \cap \mathcal{B}\left([D(h)]^{*}, L^{2}\left(\mathbb{R}^{n}\right)\right)
$$

$($ see b)) and

$$
[H, \varphi] \in \mathcal{B}\left(D(h), L^{2}\left(\mathbb{R}^{n}\right)\right) \cap \mathcal{B}\left(L^{2}\left(\mathbb{R}^{n}\right),[D(h)]^{*}\right)
$$

(see $(2.11))$.

e) The property follows from b) and the fact that $[H, \varphi] \in \mathcal{B}\left(D(h), L^{2}\left(\mathbb{R}^{n}\right)\right)$.

f) The statement follows from $\left(H_{\Omega}+\lambda\right)^{-1} \in \mathcal{B}\left(L^{2}(\Omega), D\left(h_{\Omega}\right)\right)$ and $\partial_{k}-\mathrm{i} a_{k} \in$ $\mathcal{B}\left(D\left(h_{\Omega}\right), L^{2}(\Omega)\right)$, where $D\left(h_{\Omega}\right)$ is endowed with the norm induced by the one of $D(h)$.

We shall also need to write under a certain form the difference between the resolvent of $H$ and the pseudo-resolvent of $H_{\Omega}$.

Lemma 3.2. Let $\lambda>-\inf \sigma(H)$ and $\varphi$ be a function as in Lemma 2.7, $\varphi=1$ on $\mathbb{R}^{n} \backslash \Omega$. Then

$$
\begin{aligned}
(H+\lambda)^{-1} & -\left(H_{\Omega}+\lambda\right)^{-1} \\
& =\left[(H+\lambda)^{-1}-\left(H_{\Omega}+\lambda\right)^{-1}\right]\left[\varphi+[H, \varphi]\left(H_{\Omega}+\lambda\right)^{-1}\right] \\
& =\left[\varphi-(H+\lambda)^{-1}[H, \varphi]\right]\left[(H+\lambda)^{-1}-\left(H_{\Omega}+\lambda\right)^{-1}\right]
\end{aligned}
$$


Proof. We have

$$
\begin{aligned}
(H+\lambda)^{-1}- & \left(H_{\Omega}+\lambda\right)^{-1}=s-\lim _{\alpha \rightarrow \infty}\left[(H+\lambda)^{-1}-\left(H_{\alpha}+\lambda\right)^{-1}\right] \\
= & s-\lim _{\alpha \rightarrow \infty}(H+\lambda)^{-1} \alpha\left(1-P_{\Omega}\right)\left(H_{\alpha}+\lambda\right)^{-1} \\
= & s-\lim _{\alpha \rightarrow \infty}(H+\lambda)^{-1} \alpha\left(1-P_{\Omega}\right) \varphi\left(H_{\alpha}+\lambda\right)^{-1} \\
= & s-\lim _{\alpha \rightarrow \infty}\left[(H+\lambda)^{-1} \alpha\left(1-P_{\Omega}\right)\left(H_{\alpha}+\lambda\right)^{-1} \varphi\right. \\
& \left.\quad+(H+\lambda)^{-1} \alpha\left(1-P_{\Omega}\right)\left(H_{\alpha}+\lambda\right)^{-1}[H, \varphi]\left(H_{\alpha}+\lambda\right)^{-1}\right] \\
= & {\left[(H+\lambda)^{-1}-\left(H_{\Omega}+\lambda\right)^{-1}\right] \varphi } \\
& \quad+\left[(H+\lambda)^{-1}-\left(H_{\Omega}+\lambda\right)^{-1}\right][H, \varphi]\left(H_{\Omega}+\lambda\right)^{-1},
\end{aligned}
$$

where in the last equality we have used the property e) from Proposition 3.1, as well as the fact that $\left(H_{\alpha}+\lambda\right)^{-1}$ is bounded in $\mathcal{B}\left(L^{2}\left(\mathbb{R}^{n}\right)\right)$ uniformly with respect to $\alpha \geq 0$.

In the same way we prove the equality between the first and the last term of relation (3.1).

Now we can generalize the inequality (2.1) to the operator $H_{\Omega}$.

Proposition 3.3. Under the hypotheses i) and ii), for every $\rho>1$ there exist $M, \delta>0$ such that if $\lambda>\max \{\delta,-\inf \sigma(H)\}$, we have

$$
\left|\left(H_{\Omega}+\lambda\right)^{-r}\right| \leq M P_{\Omega}\left(\rho H_{0}+\lambda-\delta\right)^{-r} P_{\Omega}|f| \text { a.e on } \mathbb{R}^{n},
$$

for every $r>0, \Omega$ open subset of $\mathbb{R}^{n}$ and $f \in L^{2}\left(\mathbb{R}^{n}\right)$.

Proof. Using Proposition 3.1 a), we see that for every $t>0$

$$
s-\lim _{\alpha \rightarrow 0} \mathrm{e}^{-t H_{\alpha}}=\mathrm{e}^{-t H_{\Omega}} .
$$

We also note that the Feymann-Kaç formula allows us to derive the inequality

$$
\mathrm{e}^{-t H\left(0, V+\alpha\left(1-\chi_{\Omega}\right)\right)}|f| \leq \mathrm{e}^{-t H\left(0,-V_{-}\right)}|f| \text { a.e. on } \mathbb{R}^{n} .
$$

Hence, using (3.3), (3.4) and the first part of the proof of Proposition 2.1, we get

$$
\left|\mathrm{e}^{-t H_{\Omega}} f\right| \leq M \mathrm{e}^{\delta t} \mathrm{e}^{-\rho t H_{0}}|f| \text { a.e. on } \mathbb{R}^{n} .
$$

We infer from Proposition 3.1 a) that, for every $r>0$,

$$
s-\lim _{\alpha \rightarrow 0}\left(H_{\alpha}+\lambda\right)^{-r}=\left(H_{\Omega}+\lambda\right)^{-r} .
$$


Hence, from the equality (2.5) for $H_{\alpha}$, from (3.5) and (3.6), it follows that

$$
\left|\left(H_{\Omega}+\lambda\right)^{-r} f\right| \leq M\left(\rho H_{0}+\lambda-\delta\right)^{-r}|f| \text { a.e. on } \mathbb{R}^{n} \text {. }
$$

To get (3.2) it suffices to write (3.7) for $P_{\Omega} f$ instead of $f$ and to use Proposition $3.1 \mathrm{~b})$.

The proof of Proposition 2.4, with (2.1) replaced by (3.2), allows us to obtain the next proposition.

Proposition 3.4. $\quad$ Under the hypotheses of Proposition 3.3, for every $r>n / 4$ and $\lambda>\max \{\delta,-\inf \sigma(H)\}$, there exists a positive constant $C$ such that for every $U$ and $\Omega$ open subsets of $\mathbb{R}^{n}, U$ bounded, we have that $P_{U}\left(H_{\Omega}+\right.$ $\lambda)^{-r} \in \mathcal{I}_{2}$ and the inequality

$$
\left\|P_{U}\left(H_{\Omega}+\lambda\right)^{-r}\right\|_{\mathcal{I}_{2}} \leq C|\Omega \cap U|^{1 / 2}
$$

holds.

The next two corollaries follow directly from the proposition above (see the proofs of Corollary 2.5 and 2.6).

Corollary 3.5. Under the hypotheses of Proposition 3.3, for every $m>$ $n / 2$ and $\lambda>\max \{\delta,-\inf \sigma(H)\}$, there exists a positive constant $C$ such that for every $\Omega$ open bounded subset of $\mathbb{R}^{n}$, we have that $\left(H_{\Omega}+\lambda\right)^{-m} \in \mathcal{I}_{1}$ and the inequality

$$
\left\|\left(H_{\Omega}+\lambda\right)^{-m}\right\|_{\mathcal{I}_{1}} \leq C|\Omega|
$$

holds.

Corollary 3.6. For every $f \in L_{\text {comp }}^{\infty}(\mathbb{R})$ there exists a constant $C>0$ such that, for every $\Omega$ open bounded subset of $\mathbb{R}^{n}$, we have that $f\left(H_{\Omega}\right) \in \mathcal{I}_{1}$ and the inequality

$$
\left\|f\left(H_{\Omega}\right)\right\|_{\mathcal{I}_{1}} \leq C|\Omega|
$$

holds.

The last result of this section will be the equality $H_{\Omega}=\tilde{H}_{\Omega}$ for open subsets of $\mathbb{R}^{n}$ with minimally smooth boundary. This equality is a consequence of the following proposition. 
Proposition 3.7. $\quad$ Let $\Omega$ be an open subset of $\mathbb{R}^{n}$ with minimally smooth boundary (cf. Stein [15]). Then $\mathcal{C}_{0}^{\infty}(\Omega)$ is a core of the sesqui-linear form $h_{\Omega}$.

Proof. The proof is divided in three steps, in each of them obtaining partial results.

i) $D\left(h_{\Omega}\right) \cap L^{\infty}(\Omega)$ is a core of $h_{\Omega}$.

We use an idea from [11]. It is obvious that the range of $\mathrm{e}^{-H_{\Omega}}$ is a core of $H_{\Omega}$, hence also for $h_{\Omega}$. On the other hand, for every $\rho>0, \mathrm{e}^{-\rho H_{0}}$ is a convolution operator by an $L^{2}\left(\mathbb{R}^{n}\right)$-function, hence $\mathrm{e}^{-\rho H_{0}} \in \mathcal{B}\left(L^{2}\left(\mathbb{R}^{n}\right), L^{\infty}\left(\mathbb{R}^{n}\right)\right)$. The inequality (3.5) implies therefore that the range of $\mathrm{e}^{-H_{\Omega}}$ is contained in $L^{\infty}(\Omega)$.

ii) $D\left(h_{\Omega}\right) \cap L_{\text {comp }}^{\infty}(\Omega)$ is a core of $h_{\Omega}$.

There exist (see [15]) $N \in \mathbb{N}$, a sequence $\left(\Omega_{i}\right)_{i \geq 1}$ of open subsets of $\mathbb{R}^{n}$ and two sequences of functions $\left(\varphi_{i}\right)_{i \geq 1}$ and $\left(\psi_{i}\right)_{i \geq 0}$ with the following properties:

1. $\partial \Omega \subset \bigcup_{i \geq 1} \Omega_{i}$.

2. The intersection of $N+1$ open sets $\Omega_{i}$ is void.

3. The functions $\varphi_{i}: \mathbb{R}^{n-1} \rightarrow \mathbb{R}$ are Lipschitz and the sequence of their Lipschitz constants is bounded.

4. We may suppose that $\Omega \cap \Omega_{i}=\left\{x \in \Omega_{i} ; x_{n}>\varphi_{i}\left(x^{\prime}\right)\right\}, i \geq 1$, where $x=\left(x^{\prime}, x_{n}\right) \in \mathbb{R}^{n-1} \times \mathbb{R}=\mathbb{R}^{n}$.

5. $\psi_{i} \in \mathcal{C}^{\infty}\left(\mathbb{R}^{n}\right), \psi_{i} \geq 0, \operatorname{supp} \psi_{0} \in \Omega$, and $\operatorname{supp} \psi_{i} \in \Omega_{i}$ for $i \geq 1$.

6. For every $\alpha \in \mathbb{N}^{n}, \partial^{\alpha} \psi_{i}$ are bounded uniformly with respect to $i \geq 0$.

7. $\sum_{i \geq 0} \psi_{i}=1$ on $\bar{\Omega}$.

Let $u \in D\left(h_{\Omega}\right) \cap L^{\infty}(\Omega)$. Then $\operatorname{supp} u \subset \bar{\Omega}$ and $u=\sum_{i \geq 0} \psi_{i} u$; the series converges in $D\left(h_{\Omega}\right)$, by the dominated convergence theorem. It suffices to prove that for every $i \geq 0, \psi_{i} u$ is the limit in $D\left(h_{\Omega}\right)$ of a sequence from $D\left(h_{\Omega}\right) \cap L_{\text {comp }}^{\infty}(\Omega)$. We can construct a partition of unity on a neighborhood of $\operatorname{supp}\left(\psi_{i} u\right)$, that is a sequence $\left(\beta_{j}\right)_{j \geq 1}$, with $\beta_{j} \in \mathcal{C}_{0}^{\infty}\left(\mathbb{R}^{n}\right), \beta_{j} \geq 0$, the family $\left(\operatorname{supp} \beta_{j}\right)_{j \geq 0}$ being locally finite and $\sum_{j \geq 0} \beta_{j}=1$ on a neighborhood of $\operatorname{supp}\left(\psi_{i} u\right)$. We may also suppose that for every $\alpha \in \mathbb{N}^{n}$, the sequence $\left(\partial^{\alpha} \beta_{j}\right)_{j \geq 0}$ is uniformly bounded. Then $\psi_{i} u=\sum_{j \geq 0} \beta_{j}\left(\psi_{i} u\right)$, the series being 
convergent in $D\left(h_{\Omega}\right)$. It then follows that we may henceforth suppose $\psi_{i} u$ to be compactly supported.

We have that $\psi_{0} u \in D\left(h_{\Omega}\right) \cap L_{\text {comp }}^{\infty}(\Omega)$; hence it remains to show that for every $v \in D\left(h_{\Omega}\right) \cap L^{\infty}(\Omega)$, whose support is a compact subset of $\bar{\Omega} \cap \Omega_{i}$, is the limit in $D\left(h_{\Omega}\right)$ of a sequence from $D\left(h_{\Omega}\right) \cap L_{\text {comp }}^{\infty}(\Omega)$.

Let $\chi_{i}: \mathbb{R}^{n} \rightarrow \mathbb{R}^{n}$ be the homeomorphism defined by $y=\chi_{i}(x)$ if and only if $y^{\prime}=x^{\prime}, y_{n}=x_{n}-\varphi\left(x^{\prime}\right)$. It is clear that $v \in \mathcal{H}_{\text {comp }}^{1}\left(\mathbb{R}^{n}\right)$ (the Sobolev space of order 1 on $\mathbb{R}^{n}$, whose elements are compactly supported). Then $w:=v \circ \chi_{i}^{-1}$ belongs to $\mathcal{H}_{\text {comp }}^{1}\left(\mathbb{R}^{n}\right)$ and $\operatorname{supp} w \subset \overline{\mathbb{R}_{+}^{n}}$. We consider a function $\theta \in \mathcal{C}_{0}^{\infty}\left(\mathbb{R}^{n}\right)$, $\theta \geq 0, \int_{\mathbb{R}^{n}} \theta(y) \mathrm{d} y=1$, and such that $|y| \leq 1$ and $y_{n}>0$ on $\operatorname{supp} \theta$. For $0<\varepsilon \leq 1$ we define $\theta_{\varepsilon} \in \mathcal{C}_{0}^{\infty}\left(\mathbb{R}^{n}\right)$ by $\theta_{\varepsilon}(y):=\varepsilon^{-n} \theta(y / \varepsilon)$. Let $w_{\varepsilon}$ be the convolution of $w$ by $\theta_{\varepsilon}$. Then $w_{\varepsilon} \in \mathcal{C}_{0}^{\infty}\left(\mathbb{R}^{n}\right), \operatorname{supp} w_{\varepsilon} \subset \mathbb{R}_{+}^{n}, \sup _{0<\varepsilon \leq 1}\left\|w_{\varepsilon}\right\|_{L^{\infty}\left(\mathbb{R}^{n}\right)}<\infty$ and $\lim _{\varepsilon \searrow 0} w_{\varepsilon}=w$ in $\mathcal{H}^{1}\left(\mathbb{R}^{n}\right)$.

If $v_{\varepsilon}:=w_{\varepsilon} \circ \chi_{i}$, then $v_{\varepsilon} \in \mathcal{H}_{\text {comp }}^{1}\left(\mathbb{R}^{n}\right), \sup _{0<\varepsilon \leq 1}\left\|v_{\varepsilon}\right\|_{L^{\infty}\left(\mathbb{R}^{n}\right)}<\infty, \lim _{\varepsilon \backslash 0} v_{\varepsilon}$ $=v$ in $\mathcal{H}^{1}\left(\mathbb{R}^{n}\right)$, and there exist $\varepsilon_{0} \in(0,1]$ and a compact $K$ contained in $\bar{\Omega} \cap \Omega_{i}$ such that $\operatorname{supp} v_{\varepsilon} \subset K \cap \Omega$ for all $0<\varepsilon \leq \varepsilon_{0}$. It is clear that $v_{\varepsilon} \in$ $D\left(h_{\Omega}\right) \cap L_{\text {comp }}^{\infty}(\Omega)$ and we easily infer the existence of a sequence $\left(\varepsilon_{j}\right)_{j \geq 0}$, $0<\varepsilon_{j} \leq \varepsilon_{0}, \lim _{j \rightarrow \infty} \varepsilon_{j}=0$ such that $\lim _{j \rightarrow \infty} v_{\varepsilon_{j}}=v$ in $D\left(h_{\Omega}\right)$.

iii) $\mathcal{C}_{0}^{\infty}(\Omega)$ is a core of $h_{\Omega}$.

Let $u \in D\left(h_{\Omega}\right) \cap L_{\text {comp }}^{\infty}(\Omega)$ and $\left(\theta_{\varepsilon}\right)_{0<\varepsilon \leq \varepsilon_{0}}$ be the sequence constructed in ii). If $\varepsilon_{0}$ is small enough, $u_{\varepsilon}:=u * \theta_{\varepsilon} \in \mathcal{C}_{0}^{\infty}(\Omega)$ and there exists a compact subset $M$ of $\Omega$ such that $\operatorname{supp} u_{\varepsilon} \subset M$ for all $\varepsilon \in\left(0, \varepsilon_{0}\right]$. Moreover, the sequence $\left(u_{\varepsilon}\right)_{0<\varepsilon \leq \varepsilon_{0}}$ is uniformly bounded and $\lim _{\varepsilon \backslash 0} u_{\varepsilon}=u$ in $\mathcal{H}^{1}(\Omega)$, since $u \in \mathcal{H}^{1}(\Omega)$. Hence, there exists a sequence $\left(\varepsilon_{j}\right)_{j \geq 0}, 0<\varepsilon_{j} \leq \varepsilon_{0}, \lim _{j \rightarrow \infty} \varepsilon_{j}=0$ such that $\lim _{j \rightarrow \infty} u_{\varepsilon_{j}}=u$ in $D\left(h_{\Omega}\right)$.

\section{$\S 4 . \quad$ Proof of Theorem 1.4}

The main ingredient of the proof will be the following result.

Proposition 4.1. Under the hypotheses of Proposition 3.3, for every $m \in \mathbb{N}, m \geq n+2$ and $\lambda>\max \{\delta,-\inf \sigma(H)\}$, there exists a positive constant $C$ such that we have

$$
\left\|P_{\Omega}(H+\lambda)^{-m} P_{\Omega}-\left(H_{\Omega}+\lambda\right)^{-m}\right\|_{\mathcal{I}_{1}} \leq C|\Omega|^{1 / 2}|\tilde{\Omega}|^{1 / 2}
$$

for every $\Omega$ bounded open subset of $\mathbb{R}^{n}$, where $\tilde{\Omega}:=\{x \in \Omega$; $\operatorname{dist}(x, \partial \Omega)<1\}$. 
Proof. We have the identity

$$
\begin{aligned}
& P_{\Omega}(H+\lambda)^{-m} P_{\Omega}-\left(H_{\Omega}+\lambda\right)^{-m}=P_{\Omega}\left[(H+\lambda)^{-m}-\left(H_{\Omega}+\lambda\right)^{-m}\right] P_{\Omega} \\
& =\sum_{j=0}^{m-1} P_{\Omega}(H+\lambda)^{j-m+1}\left[(H+\lambda)^{-1}-\left(H_{\Omega}+\lambda\right)^{-1}\right]\left(H_{\Omega}+\lambda\right)^{-j} P_{\Omega} .
\end{aligned}
$$

Let $\varphi \in \mathcal{C}^{\infty}\left(\mathbb{R}^{n}\right)$ be such that $\varphi=1$ on $\mathbb{R}^{n} \backslash \Omega, \varphi=0$ on $\Omega \backslash \tilde{\Omega}$, and for every $\alpha \in \mathbb{N}^{n}, \partial^{\alpha} \varphi$ is bounded by a constant independent on $\Omega$ (we may construct it by considering the convolution of the characteristic function of a neighborhood of $\mathbb{R}^{n} \backslash \Omega$ by a appropriate function from $\mathcal{C}_{0}^{\infty}\left(\mathbb{R}^{n}\right)$ ).

We use Propositions 3.4, $3.1 \mathrm{~d}$ ), Lemma 2.7 and the first equality in (3.1) to estimate the $\mathcal{I}_{1}$-norm of the terms in the sum in (4.2) corresponding to $j>n / 2$. Everything reduces to the following two estimates:

$$
\begin{aligned}
& \left\|P_{\Omega}(H+\lambda)^{j-m+1}\left[(H+\lambda)^{-1}-\left(H_{\Omega}+\lambda\right)^{-1}\right] \varphi\left(H_{\Omega}+\lambda\right)^{-j-1} P_{\Omega}\right\|_{\mathcal{I}_{1}} \\
& \quad \leq C_{1}\left\|\varphi\left(H_{\Omega}+\lambda\right)^{-j / 2}\right\|_{\mathcal{I}_{2}}\left\|\left(H_{\Omega}+\lambda\right)^{-j / 2} P_{\Omega}\right\|_{\mathcal{I}_{2}} \leq C_{2}|\Omega|^{1 / 2}|\tilde{\Omega}|^{1 / 2}
\end{aligned}
$$

and

$$
\begin{aligned}
& \left\|P_{\Omega}(H+\lambda)^{j-m+1}\left[(H+\lambda)^{-1}-\left(H_{\Omega}+\lambda\right)^{-1}\right][H, \varphi]\left(H_{\Omega}+\lambda\right)^{-j-1} P_{\Omega}\right\|_{\mathcal{I}_{1}} \\
& \quad \leq C_{3}\left\|P_{\tilde{\Omega}}\left(H_{\Omega}+\lambda\right)^{-j-1}\right\|_{\mathcal{I}_{1}} \leq C_{4}|\Omega|^{1 / 2}|\tilde{\Omega}|^{1 / 2}
\end{aligned}
$$

where the constants $C_{j}, 1 \leq j \leq 4$, do not depend on $\Omega$, and we should consider the fact that the derivatives of $\varphi$ have supports contained in $\tilde{\Omega}$.

The terms with $j \leq n / 2$ (hence $m-j-1>n / 2$ ) are estimated in the same way, using the other equality of (3.1) and the identity $\varphi(H+\lambda)^{-1}=$ $(H+\lambda)^{-1} \varphi+(H+\lambda)^{-1} H, \varphi(H+\lambda)^{-1}$.

The assertions of Theorem 1.4 will follow from the next proposition.

Proposition 4.2. $\quad$ Suppose that hypotheses i)-iv) hold. Then, for every $f \in \mathcal{C}_{0}(\mathbb{R})$ and $\varepsilon>0$, there exists $m_{0} \in \mathbb{N}^{*}$ such that we have

$$
\left|\operatorname{Tr}\left(P_{\Omega} f(H) P_{\Omega}\right)-\operatorname{Tr} f\left(H_{\Omega}\right)\right| \leq \varepsilon|\Omega|
$$

for every $\Omega \in \mathcal{F}$ with $B\left(0 ; m_{0}\right) \subset \Omega$.

Proof. The proof follows [5]. For a fixed $\rho>1$, we consider $\delta>0$ as in Proposition 3.3, and let $a:=\max \{\delta,-\inf \sigma(H)\}+1$. It suffices to prove (4.3) for real functions $f$ with supp $f \subset[-a+1 / 2, \infty)$. The functions $[-a+1 / 2, \infty) \ni$ 
$t \rightarrow(a+t)^{n+2} f(t) \in \mathbb{R}$ and $[0,2] \ni \tau \rightarrow \tau^{-n-2} f\left(\tau^{-1}-a\right) \in \mathbb{R}$ are continuous.

For every $\varepsilon>0$ there exists a polynomial $P_{\varepsilon}$ such that

$$
\left|\tau^{-n-2} f\left(\tau^{-1}-a\right)-P_{\varepsilon}(\tau)\right| \leq \varepsilon \quad \text { for } \quad 0 \leq \tau \leq 2
$$

Then

$$
\left|(a+t)^{n+2} f(t)-P_{\varepsilon}\left(\frac{1}{a+t}\right)\right| \leq \varepsilon \quad \text { for } \quad t \geq-a+1 / 2 .
$$

Let $Q_{\varepsilon}(t):=(a+t)^{-n-2} P_{\varepsilon}\left(\frac{1}{a+t}\right)$. Then, in form sense,

$$
-\varepsilon(a+H)^{-n-2} \leq f(H)-Q_{\varepsilon}(H) \leq \varepsilon(a+H)^{-n-2},
$$

hence

$$
\begin{aligned}
-\varepsilon P_{\Omega}(a+H)^{-n-2} P_{\Omega} & \leq P_{\Omega} f(H) P_{\Omega}-P_{\Omega} Q_{\varepsilon}(H) P_{\Omega} \\
& \leq \varepsilon P_{\Omega}(a+H)^{-n-2} P_{\Omega} .
\end{aligned}
$$

Using Corollary 2.5 we infer

$$
\begin{aligned}
& \left|\operatorname{Tr}\left(P_{\Omega} f(H) P_{\Omega}\right)-\operatorname{Tr}\left(P_{\Omega} Q_{\varepsilon}(H) P_{\Omega}\right)\right| \\
& \quad \leq \varepsilon \operatorname{Tr}\left(P_{\Omega}(a+H)^{-n-2} P_{\Omega}\right) \leq C_{1} \varepsilon|\Omega|,
\end{aligned}
$$

where $C_{1}$ is a constant independent on $\varepsilon$ and $\Omega \in \mathcal{F}$.

Similarly we prove that there exists another constant $C_{2}$, independent on $\varepsilon$ and $\Omega \in \mathcal{F}$, such that we have

$$
\left|\operatorname{Tr} f\left(H_{\Omega}\right)-\operatorname{Tr} Q_{\varepsilon}\left(H_{\Omega}\right)\right| \leq \varepsilon \operatorname{Tr}\left(a+H_{\Omega}\right)^{-n-2} \leq C_{2} \varepsilon|\Omega| .
$$

Therefore (4.3) follows from (4.4), (4.5), Proposition 4.1 and hypothesis iv).

\section{$\S 5 . \quad$ Proof of Theorem 1.6}

We shall identify the $\Gamma$-periodic distributions on $\mathbb{R}^{n}$ to the distributions on the torus $\mathbb{T}^{n}=\mathbb{R}^{n} / \Gamma$. The duality bracket for the dual pair $\left(\mathcal{D}^{\prime}\left(\mathbb{T}^{n}\right), \mathcal{D}\left(\mathbb{T}^{n}\right)\right)$ is denoted by $\langle\cdot, \cdot\rangle_{\Gamma}$, while $\langle\cdot, \cdot\rangle$ is the scalar product of $\mathbb{R}^{n}$. Let

$$
\Gamma^{*}=\left\{\gamma^{*} \in \mathbb{R}^{n} ;\left\langle\gamma^{*}, \gamma\right\rangle \in 2 \pi \mathbb{Z} \text { for every } \gamma \in \Gamma\right\}
$$

be the dual lattice of $\Gamma$.

Proposition 5.1. Let $B=\frac{1}{2} \sum_{1 \leq j, k \leq n} B_{j k} \mathrm{~d} x_{j} \wedge \mathrm{d} x_{k}$ be a differential 2 -form whose coefficients $B_{j k}=-B_{k j}$ are real $\Gamma$-periodic distributions on 
$\mathbb{R}^{n}$, and such that $\mathrm{d} B=0$. Then, there exists a differential 1-form $A=$ $\sum_{1 \leq j \leq n} A_{j} \mathrm{~d} x_{j}$, with coefficients $A_{j}$ real $\Gamma$-periodic distributions on $\mathbb{R}^{n}$ and such that $\mathrm{d} A=B$, if and only if

$$
\left\langle B_{j k}, 1\right\rangle_{\Gamma}=0, \quad 1 \leq j, k \leq n .
$$

Moreover, if the coefficients $B_{j k}$ belong to the Sobolev space $\mathcal{H}^{-1}\left(\mathbb{T}^{n}\right), 1 \leq$ $j, k \leq n$, we can choose $A_{j} \in L^{2}\left(\mathbb{T}^{n}\right), 1 \leq j \leq n$.

Proof. We may write

$$
B_{j k}=\sum_{\alpha \in \Gamma^{*}} B_{\alpha}^{j k} \mathrm{e}^{\mathrm{i}\langle\cdot, \alpha\rangle}, \quad B_{\alpha}^{j k}:=\frac{1}{|F|}\left\langle B_{j k}, \mathrm{e}^{-\mathrm{i}\langle\cdot, \alpha\rangle}\right\rangle_{\Gamma},
$$

the series being convergent in $\mathcal{D}^{\prime}\left(\mathbb{T}^{n}\right)$, which means that there exists a constant $C>0$ and $p \in \mathbb{Z}$ such that we have

$$
\left|B_{\alpha}^{j k}\right| \leq C(1+|\alpha|)^{p}, \quad \alpha \in \Gamma^{*}, 1 \leq j, k \leq n .
$$

The condition $\mathrm{d} B=0$ means that $\partial_{l} B_{j k}+\partial_{k} B_{l j}+\partial_{j} B_{k l}=0$, hence

$$
\alpha_{l} B_{\alpha}^{j k}+\alpha_{k} B_{\alpha}^{l j}+\alpha_{j} B_{\alpha}^{k l}=0, \quad 1 \leq j, k, l \leq n,
$$

where $\alpha=\left(\alpha_{1}, \ldots \alpha_{n}\right)$. Similarly, we may represent $A$ in the form

$$
A_{j}=\sum_{\alpha \in \Gamma^{*}} A_{\alpha}^{j} \mathrm{e}^{\mathrm{i}\langle\cdot, \alpha\rangle}, \quad A_{\alpha}^{j}:=\frac{1}{|F|}\left\langle A_{j}, \mathrm{e}^{-\mathrm{i}\langle\cdot, \alpha\rangle}\right\rangle_{\Gamma},
$$

and we have to find $C>0, q \in \mathbb{Z}$ such that

$$
\left|A_{\alpha}^{j}\right| \leq C(1+|\alpha|)^{q}, \quad \alpha \in \Gamma^{*}, 1 \leq j \leq n .
$$

The equation $\mathrm{d} A=B$, that is, the differential system

$$
\partial_{j} A_{k}-\partial_{k} A_{j}=B_{j k}, \quad 1 \leq j, k \leq n,
$$

is equivalent to the algebraic system

$$
\alpha_{j} A_{\alpha}^{k}-\alpha_{k} A_{\alpha}^{j}=-\mathrm{i} B_{\alpha}^{j k}, \quad 1 \leq j, k, l \leq n, \alpha \in \Gamma^{*} .
$$

The condition (5.1) means $B_{0}^{j k}=0,1 \leq j, k \leq n$, and it is a necessary condition for the existence of a solution to the system (5.6). Considering (5.3), it is also sufficient, since the general solution to (5.6) is

$$
A_{\alpha}^{j}= \begin{cases}C_{0} & \text { for } \alpha=0 \\ -\mathrm{i}|\alpha|^{-2} \sum_{1 \leq k \leq n} \alpha_{k} B_{\alpha}^{k j}+\mathrm{i} \alpha_{j} C_{\alpha} & \text { for } \alpha \neq 0\end{cases}
$$


where $C_{\alpha}$ are arbitrary constants with $\overline{C_{\alpha}}=C_{-\alpha}$. If we choose $C_{\alpha}=0$ for $\alpha \neq 0$, we may take $q=p-1$ in (5.5). We have $\overline{B_{\alpha}^{k j}}=B_{-\alpha}^{k j}$, therefore also $\overline{A_{\alpha}^{j}}=A_{-\alpha}^{j}$, and the distribution $A_{j}$ is real.

To prove the last assertion, it suffices to note the fact that $B_{j k} \in \mathcal{H}^{-1}\left(\mathbb{T}^{n}\right)$ means

$$
\sum_{\alpha}(1+|\alpha|)^{-2}\left|B_{\alpha}^{j k}\right|^{2}<\infty
$$

Then the solution (5.7) with $C_{\alpha}^{j}=0$ for $\alpha \neq 0$ verifies

$$
\sum_{\alpha}\left|A_{\alpha}^{j}\right|^{2}<\infty
$$

hence $A_{j} \in L^{2}\left(\mathbb{T}^{n}\right)$.

Corollary 5.2. Assume that hypotheses i), ii) and v) hold. Then there exists a vector potential $A=\sum_{1 \leq j \leq n} A_{j} \mathrm{~d} x_{j}$ with $A_{j} \in L_{\mathrm{loc}}^{2}\left(R^{n}, \mathbb{R}\right)$ and $\Gamma$ periodic, $1 \leq j \leq n$, and a constant magnetic field $B^{0}=\frac{1}{2} \sum_{1 \leq j, k \leq n} B_{j k}^{0} \mathrm{~d} x_{j} \wedge \mathrm{d} x_{k}$, $B_{j k}^{0}=-B_{k j}^{0} \in \mathbb{R}$, such that if $A^{0}=\sum_{1 \leq j \leq n} A_{j}^{0} \mathrm{~d} x_{j}$ with $A_{j}^{0}(x)=\frac{1}{2} \sum_{1 \leq k \leq n} B_{j k}^{0} x_{k}$, the operators $H(a, V)$ and $H\left(A+A_{0}, V\right)$ are unitarily equivalent.

Proof. We first choose the form $B^{0}$ with $B_{j k}^{0}=-B_{k j}^{0} \in \mathbb{R}$ such that we have $\left\langle B_{j k}-B_{j k}^{0}, 1\right\rangle_{\Gamma}=0,1 \leq j, k \leq n$. It is obvious that $B_{j k} \in \mathcal{H}^{-1}\left(\mathbb{T}^{n}\right)$, and therefore, using Proposition 5.1, we infer the existence of a 1-form $A=$ $\sum_{1 \leq j \leq n} A_{j} \mathrm{~d} x_{j}$ with $A_{j} \in L_{\text {loc }}^{2}\left(R^{n}, \mathbb{R}\right)$ and $\Gamma$-periodic, such that $\mathrm{d} A=B-B^{0}$. If $A^{0}$ is the 1 -form from the statement, we shall have $\mathrm{d}\left(A+A_{0}\right)=B=\mathrm{d} a$. Using Lemma 1.1 in [8], we deduce the existence of a real function $g \in \mathcal{H}_{\text {loc }}^{1}\left(\mathbb{R}^{n}\right)$ such that $a-\left(A+A^{0}\right)=\nabla g$. If $U$ is the multiplication operator by $\mathrm{e}^{\mathrm{i} g}$, which is unitary on $L^{2}\left(\mathbb{R}^{n}\right)$, Theorem 1.2 in [8] implies $U H(a, V) U^{-1}=H(A+$ $\left.A^{0}, V\right)$.

Remark 5.3. Considering the definition of the density of states, we see that it suffices to prove the existence of this measure for $H\left(A+A^{0}, V\right)$. Therefore we may henceforth assume that $a=A+A^{0}$.

Let $T_{\gamma}, \gamma \in \Gamma$, the magnetic translations defined by $B^{0}$ in Corollary 5.2. Hence $T_{\gamma}=U_{\gamma} L_{\gamma}$, where $L_{\gamma}$ are the usual translations in $\mathbb{R}^{n}:\left(L_{\gamma} u\right)(x)=$ $u(x-\gamma), x \in \mathbb{R}^{n}, \gamma \in \Gamma$, and $\left(U_{\gamma} u\right)(x)=\mathrm{e}^{\mathrm{i}\left\langle B^{0}, x \wedge \gamma\right\rangle / 2} u(x), B^{0}$ being viewed here as a linear form on $R^{n} \wedge \mathbb{R}^{n}$.

Using the model in [1] (even if $\left\{T_{\gamma}\right\}_{\gamma}$ is not a group) we can define a $\Gamma$-trace on a class of operators from $\mathcal{B}\left(L^{2}\left(\mathbb{R}^{n}\right)\right)$. 
Definition 5.4. An operator $S \in \mathcal{B}\left(L^{2}\left(\mathbb{R}^{n}\right)\right)$ commuting with the magnetic translations $T_{\gamma}, \gamma \in \Gamma$ is said to be of $\Gamma$-trace class if for every functions $\varphi, \psi \in L_{\text {comp }}^{\infty}\left(\mathbb{R}^{n}\right)$ we have $\varphi S \psi \in \mathcal{I}_{1}$. We write $S \in \mathcal{I}_{1}^{\Gamma}$.

Lemma 5.5. Let $S \in \mathcal{I}_{1}^{\Gamma}$ and $\varphi, \varphi^{\prime}, \psi, \psi^{\prime} \in L_{\text {comp }}^{2}\left(\mathbb{R}^{n}\right)$ such that $\sum_{\gamma \in \Gamma} L_{\gamma}(\varphi \psi)=\sum_{\gamma \in \Gamma} L_{\gamma}\left(\varphi^{\prime} \psi^{\prime}\right)=1$. Then $\operatorname{Tr}(\varphi S \psi)=\operatorname{Tr}\left(\varphi^{\prime} S \psi^{\prime}\right)$.

Proof. We have

$$
\begin{aligned}
\operatorname{Tr}(\varphi S \psi) & =\operatorname{Tr}\left(\sum_{\gamma \in \Gamma}\left[L_{\gamma}\left(\varphi^{\prime} \psi^{\prime}\right)\right] \varphi S \psi\right)=\sum_{\gamma \in \Gamma} \operatorname{Tr}\left[\left(L_{\gamma}\left(\varphi^{\prime} \psi^{\prime}\right)\right) \varphi S \psi\right] \\
& =\sum_{\gamma \in \Gamma} \operatorname{Tr}\left[\left(L_{\gamma} \varphi^{\prime}\right) \varphi \psi S\left(L_{\gamma} \psi^{\prime}\right)\right]=\sum_{\gamma \in \Gamma} \operatorname{Tr}\left[T_{\gamma}\left(\left(L_{\gamma} \varphi^{\prime}\right) \varphi \psi S\left(L_{\gamma} \psi^{\prime}\right)\right) T_{\gamma}^{-1}\right] \\
& =\sum_{\gamma \in \Gamma} \operatorname{Tr}\left(\left[L_{\gamma}^{-1}(\varphi \psi)\right] \varphi^{\prime} S \psi^{\prime}\right)=\operatorname{Tr}\left(\sum_{\gamma \in \Gamma}\left[L_{\gamma}^{-1}(\varphi \psi)\right] \varphi^{\prime} S \psi^{\prime}\right) \\
& =\operatorname{Tr}\left(\varphi^{\prime} S \psi^{\prime}\right)
\end{aligned}
$$

where one should keep in mind the fact that the sums are finite and that $T_{\gamma}$ is a unitary operator on $L^{2}\left(\mathbb{R}^{n}\right)$.

Thus, the following definition is justified.

Definition 5.6. If $S \in \mathcal{I}_{1}^{\Gamma}$, we call $\Gamma$-trace of $S$ the quantity $\operatorname{Tr}_{\Gamma} S:=$ $\operatorname{Tr}(\varphi S \psi)$, where $\varphi, \psi \in L_{\text {comp }}^{\infty}\left(\mathbb{R}^{n}\right)$ and $\sum_{\gamma \in \Gamma} L_{\gamma}(\varphi \psi)=1$.

Lemma 5.7. If $S$ is a self-adjoint operator from $\mathcal{B}\left(L^{2}\left(\mathbb{R}^{n}\right)\right)$ and $S \in$ $\mathcal{I}_{1}^{\Gamma}$, then $K_{S}$, the integral kernel of $S$, is an $L_{\text {loc }}^{1}$-function, its restriction to the diagonal of $\mathbb{R}^{n} \times \mathbb{R}^{n}$ is well-defined and locally integrable and, moreover,

$$
\operatorname{Tr}_{\Gamma} S=\int_{F} K_{S}(x, x) \mathrm{d} x .
$$

Proof. The first assertions of the statement are consequences of the following remark: if $\varphi \psi \in \mathcal{C}_{0}^{\infty}\left(\mathbb{R}^{n}\right)$, we have $\varphi S \psi \in \mathcal{I}_{1}$, therefore there exist $\Phi$, $\Psi \in \mathcal{I}_{2}$ such that $\varphi S \psi=\Phi \Psi$. But $K_{\Phi}, K_{\Psi} \in L^{2}\left(\mathbb{R}^{n} \times \mathbb{R}^{n}\right)$ and

$$
K_{\varphi S \psi}(x, y)=\int_{\mathbb{R}^{n}} K_{\Phi}(x, z) K_{\Psi}(z, y) \mathrm{d} z,
$$


and, by the Fubini Theorem, the function $\mathbb{R}^{n} \ni x \rightarrow K_{\varphi S \psi}(x, x) \in \mathbb{C}$ belongs to $L_{\text {loc }}^{1}\left(\mathbb{R}^{n}\right)$. Hence, as $K_{\varphi S \psi}=(\varphi \otimes \psi) K_{S}$, we infer that the functions $K_{S}$ and $\mathbb{R}^{n} \ni x \rightarrow K_{S}(x, x) \in \mathbb{C}$ are locally integrable.

We now take $\varphi=\psi=\chi_{F}$. Then $\sum_{\gamma \in \Gamma} L_{\gamma}(\varphi \psi)=1$, and

$$
\begin{aligned}
\operatorname{Tr}_{\Gamma} S & =\operatorname{Tr}(\varphi S \psi)=\int_{\mathbb{R}^{n}} \varphi(x) K_{S}(x, x) \mathrm{d} x \\
& =\sum_{\gamma \in \Gamma} \int_{F}\left(L_{\gamma} \varphi\right)(x) K_{S}(x+\gamma, x+\gamma) \mathrm{d} x \\
& =\int_{F} K_{S}(x, x) \mathrm{d} x .
\end{aligned}
$$

For the last equality we have used the relation $K_{S}(x+\gamma, x+\gamma)=K_{S}(x, x)$, $x \in \mathbb{R}^{n}, \gamma \in \Gamma$, a consequence of $T_{\gamma} S=S T_{\gamma}$.

Now we are able to prove Theorem 1.6, having already a meaning for the integral in (1.1). It suffices to check the existence of the limit, and the relation (1.1). By Corollary 2.6, if $\Omega \in \mathcal{F}$ and $f \in \mathcal{C}_{0}(\mathbb{R})$, then $P_{\Omega} f(H) P_{\Omega} \in \mathcal{I}_{1}$ and we have

$$
\frac{\operatorname{Tr}\left(P_{\Omega} f(H) P_{\Omega}\right)}{|\Omega|}=\frac{1}{|\Omega|} \int_{\Omega} K_{f(H)}(x, x) \mathrm{d} x .
$$

Let us consider the following sets:

$$
\begin{aligned}
\mathcal{M} & :=\{\gamma \in \Gamma ;(F+\{\gamma\}) \cap \bar{\Omega} \neq \emptyset\}, \\
\partial \mathcal{M} & :=\{\gamma \in \mathcal{M} ;(F+\{\gamma\}) \cap \partial \Omega \neq \emptyset\}, \\
\Omega_{\Gamma} & :=\bigcup_{\gamma \in \mathcal{M} \backslash \partial \mathcal{M}}(F+\{\gamma\}) \subset \Omega, \\
(\partial \Omega)_{\Gamma} & :=\bigcup_{\gamma \in \partial \mathcal{M}}(F+\{\gamma\}) .
\end{aligned}
$$

We remark that in iv') the inequality

$$
\left\{x \in \mathbb{R}^{n} ; \operatorname{dist}(x, \partial \Omega)<1\right\}<\varepsilon|\Omega|
$$

may be replaced by

$$
\left\{x \in \mathbb{R}^{n} ; \operatorname{dist}(x, \partial \Omega)<a\right\}<\varepsilon|\Omega| .
$$


Indeed, let $\chi_{a}$ be the characteristic function of the set $\left\{x \in \mathbb{R}^{n}\right.$; dist $\left.(x, \partial \Omega)<a\right\}$ and $\rho_{b}$ the characteristic function of the open ball of radius $b$ and center 0 . Then

$$
\rho_{b} * \chi_{a_{1}}(x)=\int \rho_{b}(x-y) \chi_{a_{1}}(y) \mathrm{d} y \geq \omega_{a_{1}} \chi_{a_{2}}(x)
$$

whenever $b>a_{1}+a_{2}$, where $\omega_{c}$ denotes the measure of the ball centred at 0 and of radius $c$. Hence, an integration in $x$ in the above inequality will give

$$
\omega_{b}\left|\left\{x \in \mathbb{R}^{n} ; \operatorname{dist}(x, \partial \Omega)<a_{1}\right\} \geq \omega_{a_{1}}\right|\left\{x \in \mathbb{R}^{n} ; \operatorname{dist}(x, \partial \Omega)<a_{2}\right\} .
$$

Consequently we may suppose that $\operatorname{diam} F<1$, and therefore

$$
\Omega \backslash \Omega_{\Gamma} \subset(\partial \Omega)_{\Gamma} \subset \tilde{\Omega}=\left\{x \in \mathbb{R}^{n} ; \operatorname{dist}(x, \partial \Omega)<1\right\} .
$$

By hypothesis iv'), we get that for every $\varepsilon>0$, there exists $m_{0} \in \mathbb{N}^{*}$ such that if $B\left(0 ; m_{0}\right) \subset \Omega$, we have $\left|(\partial \Omega)_{\Gamma}\right|<\varepsilon|\Omega|$, and hence $\left|\Omega \backslash \Omega_{\Gamma}\right| \leq \varepsilon|\Omega|$. For such an $\Omega$, we have

$$
\frac{\operatorname{Tr}\left(P_{\Omega} f(H) P_{\Omega}\right)}{|\Omega|}=\frac{\left|\Omega_{\Gamma}\right|}{|\Omega|} \frac{1}{\left|\Omega_{\Gamma}\right|}\left[\int_{\Omega_{\Gamma}} K_{f(H)}(x, x) \mathrm{d} x+\int_{\Omega \backslash \Omega_{\Gamma}} K_{f(H)}(x, x) \mathrm{d} x\right] .
$$

The operator $H$ commutes with the magnetic translations $T_{\gamma}, \gamma \in \Gamma$, whence $T_{\gamma} f(H)=f(H) T_{\gamma}, \gamma \in \Gamma$, and then $K_{f(H)}(x+\gamma, x+\gamma)=K_{f(H)}(x, x), x \in \mathbb{R}^{n}$, $\gamma \in \Gamma$. Hence, we infer that

$$
\frac{1}{\left|\Omega_{\Gamma}\right|} \int_{\Omega_{\Gamma}} K_{f(H)}(x, x) \mathrm{d} x=\frac{1}{|F|} \int_{F} K_{f(H)}(x, x) \mathrm{d} x .
$$

We also have $\left|\Omega_{\Gamma}\right|=|\Omega|-\left|\Omega \backslash \Omega_{\Gamma}\right|$, hence

$$
1-\varepsilon \leq \frac{\left|\Omega_{\Gamma}\right|}{|\Omega|} \leq 1
$$

Finally,

$$
\begin{aligned}
\left|\int_{\Omega \backslash \Omega_{\Gamma}} K_{f(H)}(x, x) \mathrm{d} x\right| & \leq \int_{(\partial \Omega)_{\Gamma}}\left|K_{f(H)}(x, x)\right| \mathrm{d} x \\
& =\frac{\left|(\partial \Omega)_{\Gamma}\right|}{|F|} \int_{F}\left|K_{f(H)}(x, x)\right| \mathrm{d} x \\
& \leq \frac{\varepsilon|\Omega|}{|F|} \int_{F}\left|K_{f(H)}(x, x)\right| \mathrm{d} x .
\end{aligned}
$$

Now, the equality (1.1) follows directly from (5.9)-(5.12). 
Remark 5.8. We have that $f(H) \in \mathcal{I}_{1}^{\Gamma}$ and then, by Lemma 5.7, we see that

$$
\lim _{\Omega \rightarrow \mathbb{R}^{n}, \Omega \in \mathcal{F}} \frac{\operatorname{Tr}\left(P_{\Omega} f(H) P_{\Omega}\right)}{|\Omega|}=\frac{1}{|F|} \operatorname{Tr}_{\Gamma} f(H) .
$$

\section{Acknowledgements}

The author thanks to the referee for the careful reading of the manuscript and for a number a useful suggestions, which improved the present paper.

\section{References}

[1] Atiyah, M., Elliptic operators, discrete groups and von Neumann algebras, Astérisque, 32-33 (1976), 43-72.

[2] Avron, J., Herbst, I. and Simon, B., Schrödinger operators with singular magnetic fields Duke Math. J., 45 (1978), 847-883.

[3] Boutet de Monvel, A., Georgescu, V. and Soffer, A., N-body hamiltonians with hard-core interactions, Rev. Math. Phys., 6(4) (1994), 515-596.

[4] Durand, M. and Iftimie, V., Une inégalité ponctuelle pour la résolvante d'un opérateur de Schrödinger avec un champ magnétique, Rev. Roumaine Math. Pures Appl., 40 (1995), 743-749.

[5] Doi, S., Iwatsuka, A. and Mine, T., The uniqueness of the integrated density of states for the Schrödinger operators with magnetic fields, Math. Z., 237 (2001), 335-371.

[6] Helffer, B. and Sjöstrand, J., Equation de Schrödinger avec champ magnétique et équation de Harper, Lecture Notes in Phys., 345 (1989), 118-197.

[7] Leinfelder, H., Gauge invariance of Schrödinger operators and related spectral properties, J. Operator Theory, 9 (1983), 163-179.

[8] Hupfer, T., Leschke, H., Müller, P. and Warzel, S., Existence and uniqueness of the integrated density of states for Schrödinger operators with magnetic fields and unbounded random potentials, Rev. Math. Phys., 13 (2001), 1587-1581.

[9] Leinfelder, H. and Simader, C. G., Schrödinger operators with singular magnetic vector potentials, Math. Z., 176 (1981), 1-19.

[10] Simon, B., A canonical decomposition for quadratic forms with applications to monotone convergence theorems, J. Funct. Anal., 28 (1978), 377-385.

[11] Maximal and minimal Schrödinger forms, J. Operator Theory, 1 (1979), 37-47.

[12] - Trace Ideals and their Applications, London Mathematical Society Lecture Notes Series, vol. 35, Cambridge Univ. Press, 1979.

[13] - Functional Integration and Quantum Physics, Academic Press, New York, 1979.

[14] Schrödinger semigroups, Bull. Amer. Math. Soc., 7 (1982), 447-526.

[15] Stein, E., Singular Integrals and Differentiability Properties of Functions, Princeton University Press, 1970. 